Клиническая характеристика и лечение амбулаторных пациентов с хронической сердечной
недостаточностью в Московской области

\author{
Чернявская Т.К.' • Глезер М.Г. ${ }^{2}$
}

Цель - дать клиническую характеристику пациентов с хронической сердечной недостаточностью (ХСН) в Московской области и оценить соответствие текущей терапии актуальным клиническим рекомендациям.

Материал и методы. На основании информации, предоставленной 11 амбулаторно-поликлиническими организациями Московской области в декабре 2019 г., проанализированы данные об анамнезе 286 пациентов с диагнозом ХCH, включая сопутствующие заболевания, характер проведенного обследования и его результаты, а также о текущей терапии.

Результаты. В анамнезе наиболее частым заболеванием была артериальная гипертония - 95,1\%. У 53,8\% регистрировали инфаркт миокарда, у 37,8\% - сахарный диабет, у $34,6 \%$ - фибрилляцию предсердий. Реже встречались хроническая болезнь почек (18,5\%), пороки сердца (11,9\%), инсульт $(10,5 \%)$. Из сопутствующих не сердечно-сосудистых заболеваний наиболее часто регистрировали заболевания желудочно-кишечного тракта $(25,2 \%)$, хроническую обструктивную болезнь легких или бронхиальную астму (9,8\%), анемию $(5,2 \%)$. Только $8 \%$ пациентов имели одно заболевание, тогда как $72 \%$ - 2-3 заболевания и 20\% - 4 и более. В среднем у одного пациента было 2,7 диагноза. Эхокардиографическое исследование выполнено в $82,9 \%$ случаев. В среднем фракция выброса составила $51,0 \pm 10,11 \%$, фракция выброса $40 \%$ и менее была у $11,5 \%$ пациентов. Скорость клубочковой фильтрации рассчитана у $58,7 \%$ пациентов: уровень менее 60 мл/мин/1,73 м² отмечен у $35,9 \%, 30$ мл/мин/1,73 м² и ниже - у 3,6\%. Блокаторы ренин-ангиотензин-альдостероновой системы (ингибиторы ангиотензинпревращающего фермента, антагонисты рецепторов ангиотензина, сакубитрил/валсартан) получали 83,2\% пациентов, бета-адреноблокаторы - 79,0\%, антагонисты минералокортикоидных рецепторов - 53,1\%. Гликозиды назначены 6,9\%, диуретики - 51,1\% больных.
В большинстве случаев назначенные дозы были ниже рекомендованных в международных документах.

Заключение. Подтверждена необходимость увеличения приверженности врачей выполнению клинических рекомендаций в отношении обследования и лечения пациентов с XCH.

Ключевые слова: сердечная недостаточность, клинические рекомендации, госпитализация, болезнь-модифицирующие препараты, региональные особенности

Для цитирования: Чернявская ТK, Глезер МГ. Клиническая характеристика и лечение амбулаторных пациентов с хронической сердечной недостаточностью в Московской области. Альманах клинической медицины. 2021;49(2):125-131. doi: 10.18786/2072-0505-2021-49-023.

Поступила 28.03.2021; доработана 27.04.2021; принята к публикации 30.04.2021; опубликована онлайн 20.05.2021

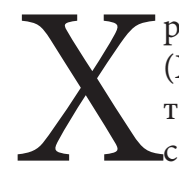
роническая сердечная недостаточность $(\mathrm{XCH})$ представляет собой одну из актуальных проблем кардиологии в связи нарастающей распространенностью среди населения, особенно в старших возрастных группах, что вносит весомый вклад в смертность от сердечно-сосудистых заболеваний и повышает количество госпитализаций [1-3]. Это обусловливает проведение интенсивных исследований по оптимизации терапии пациентов с ХCH и организационных преобразований с целью снизить смертность и оптимизировать структуру затрат [4-6]. Однако отмечаются значимые региональные различия не только в распространенности и структуре заболеваний, которые приводят к развитию ХCH, но и в характере проводимой терапии [7-10].
Московская область - второй по численности населения регион в Российской Федерации, имеющий не только городское, но и сельское население $(18,6 \%)$. При этом $24 \%$ жителей старше трудоспособного возраста [11].

Цель настоящего исследования - привести данные о клинической характеристике пациентов с ХСН в Московской области и оценить соответствие текущей терапии актуальным клиническим рекомендациям.

\section{Материал и методы}

Проведен анализ демографических и клинических характеристик пациентов, наблюдающихся в амбулаторном звене с диагнозом ХCH, в том числе данных об их анамнезе, сопутствующих заболеваниях, характере проведенного 
обследования и его результатах, а также о текущей терапии.

Медицинским организациям Московской области было предложено предоставить информацию о 10-15 пациентах с ХСН. С целью унификации собираемых показателей и дальнейшего их внесения в базу данных разработана таблица, которая включала в себя следующие параметры: возраст, пол, масса тела, функциональный класс и стадия ХСН по В.Х. Василенко и Н.Д. Стражеско, наличие в анамнезе артериальной гипертонии, инфаркта миокарда, инсульта, наличие фибрилляции предсердий, сахарного диабета (СД), хронической почечной недостаточности, пороков сердца, хронической обструктивной болезни легких и бронхиальной астмы, заболеваний желудочно-кишечного тракта, анемии. Кроме того, собирали информацию о госпитализациях по поводу сердечно-сосудистых заболеваний и их количеству за последние 12 месяцев, данные о величине артериального давления (АД), частоте сердечных сокращений, фракции выброса (ФВ), скорости клубочковой фильтрации (СКФ). Проведен учет лекарственной терапии с указанием торговых названий препаратов, суточных доз и кратности приема. Данные были собраны в течение одного месяца (декабрь 2019 г.).

Статистическая обработка данных. Использованы методы описательной статистики. Для количественных показателей, распределенных по нормальному закону, рассчитывали среднее значение и стандартное отклонение; для количественных показателей, не распределенных по нормальному закону, - среднее значение, стандартное отклонение, минимальное и максимальное значения, а также медиану и первый и третий квартили. Для качественных показателей или количественных показателей, принимающих только небольшое число возможных значений, вычисляли абсолютную и относительную частоту встречаемости каждого из возможных значений. Анализ проводили с помощью программы для работы с электронными таблицами Microsoft Office Excel.

\section{Результаты}

Собрана информация от 11 медицинских организаций Московской области, оказывающих амбулаторно-поликлиническую помощь пациентам с ХCH. Из заполненных анкет пригодными для анализа оказались 286. Возраст пациентов в сред-

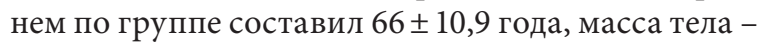
$87,7 \pm 17,2$ кг. Мужчин было 177 (61,9\%).

В анамнезе наиболее частыми заболеваниями были артериальная гипертония ( $\mathrm{n}=272 ; 95,1 \%)$,
Чернявская Татьяна Константиновна канд. мед. наук, доцент, заместитель директора по организационнометодической работе ${ }^{1}$ $\triangle 129110$, г. Москва, ул. Щепкина, 61/2, Российская Федерация. E-mail: corona1974 @mail.ru

Глезер Мария Генриховна - д-р мед. наук, профессор, профессор кафедры кардиологии, функциональной и ультразвуковой диагностики Института клинической медицины им. Н.В. СклифосовСкого ${ }^{2}$; ORCID: https:// orcid.org/0000-00020995-1924.

E-mail: 287ast@mail.ru

1 ГБУз МО «Московский областной научно-исследовательский клинический институт им. М.Ф. Владимирского»; 129110, г. Москва, ул. Щепкина, 61/2, Российская Федерация 2 ФГАОУ ВО Первый Московский государственный медицинский университет имени И.М. Сеченова Минздрава России (Сеченовский университет); 119991, г. Москва, ул. Трубецкая, 8/2, Российская Федерация перенесенный инфаркт миокарда ( $\mathrm{n}=154 ; 53,8 \%)$, СД $\quad(n=108 ; 37,8 \%)$, фибрилляция предсердий (n=99; 34,6\%). Реже встречались такие заболевания и состояния, как хроническая болезнь почек (ХБП) $(\mathrm{n}=53 ; 18,5 \%)$, пороки сердца $(\mathrm{n}=34 ; 11,9 \%)$, острое нарушение мозгового кровообращения $(\mathrm{n}=30 ; 10,5 \%)$.

Из сопутствующих не сердечно-сосудистых заболеваний у каждого четвертого пациента встречались те или иные заболевания желудочно-кишечного тракта $(\mathrm{n}=72 ; 25,2 \%)$, хроническая обструктивная болезнь легких или бронхиальная астма $(\mathrm{n}=28 ; 9,8 \%)$, анемия $(\mathrm{n}=15 ; 5,2 \%)$. На рис. 1 представлено распределение пациентов по количеству заболеваний, встречающихся у одного пациента. Видно, что лишь $8 \%$ имели одно заболевание, в то время как у 72\% наблюдались 2-3 заболевания и $20 \%$ имели по 4 и более диагнозов. Таким образом, у одного пациента в среднем было 2,7 диагноза, из них 1,72 диагноза сердечно-сосудистых заболеваний и 1 не сердечно-сосудистый диагноз.

При оценке параметров гемодинамики установлено, что в среднем по группе пациентов с ХCH систолическое АД составило 130,9 \pm 15,1 мм рт. ст., диастолическое АД - 79,4 99,6 мм рт. ст. (рис. 2). Частота сердечных сокращений в среднем составила $72,6 \pm 10,9$ удара в минуту.

Эхокардиографическое исследование было проведено 237 пациентам, что составило 82,9\%. В среднем ФВ составила 51,0 $10,11 \%$. Доля пациентов с ФВ менее 50\% была 39\%, при этом лишь 33 (11,5\%) пациента имели ФВ 40\% и менее.

СКФ рассчитана у 168 (58,7\%) пациентов, ее средние значения по группе были $63,8 \pm 15,9$ мл/мин/1,73 м². СКФ менее 60 мл/мин/1,73 м² имели 35,9\%, и у 6 (3,6\%) человек СКФ была 30 мл/мин/1,73 м² и ниже.

За последние 12 месяцев 201 (70,3\%) пациент госпитализировался по поводу сердечно-сосудистых заболеваний. Частота повторных госпитализаций составила $12,4 \%$.

Анализ терапии, проводимой на амбулаторном этапе, показал, что блокаторы ренин-ангиотензин-альдостероновой системы (РААС) (ингибиторы ангиотензинпревращающего фермента (иАПФ), антагонисты рецепторов ангиотензина, сакубитрил/валсартан) получали 83,2\% пациентов, бета-адреноблокаторы - 79,0\%, антагонисты минералокортикоидных рецепторов (АМКР) $53,1 \%$. Гликозиды получали 6,9\% и диуретики $51,1 \%$ пациентов.

В группе блокаторов РААС иАПФ получали $170(71,4 \%)$ из 238 человек, сартаны - 57 (23,9\%), 
сакубитрил/валсартан - 11 (4,6\%) пациентов. При этом среди иАПФ почти половина пациентов получала эналаприл, треть - периндоприл и каждый пятый - лизиноприл (таблица). Остальные иАПФ составляли малую долю.

Из числа пациентов, получавших сартаны, $3 / 4$ получали лозартан и $14 \%$ валсартан (см. таблицу). При анализе использованных дозировок выявлено, что в среднем доза эналаприла составляла $13,2 \pm 8,9$, лизиноприла - $13,6 \pm 9,3$, периндоприла аргинина - 5,9 2,7 и периндоприла эрбумина - 5,1 2,1 мг в сутки. Периндоприла аргинин $26,7 \%$ пациентов получали в полной дозе 10 мг, 56,7\% - в дозе 5 мг и 16,7\% - в дозе 2,5 мг. Периндоприла эрбумин 7 (35\%) человек получали в полной рекомендованной дозе 8 мг, 10 (50\%) по 4 мг, оставшиеся 15\% - 2 мг. Доза лозартана составила в среднем $62,2 \pm 28,4$, валсартана $184,4 \pm 102,8$ мг в сутки. Из 11 пациентов, получавших сакубитрил/валсартан, только трое получали препарат в рекомендованной дозе 200 мг 2 раза в день, 5 человек - в начальной дозировке 100 мг 2 раза в день, 1 - в сниженной дозе 50 мг в сутки и 2 получали однократно при рекомендованном режиме дозирования 2 раза в сутки.

Частота назначения бета-адреноблокаторов (см. таблицу) была следующей: 3/4 пациентов получали бисопролол, около 5\% - карведилол, каждый пятый - метопролол (в большинстве случаев метопролола тартрат). Еще 10 человек получали соталол в качестве противорецидивного препарата при пароксизмальной форме фибрилляции предсердий.

Как видно из таблицы, частота назначения петлевых диуретиков составила 73,8\%, при этом наибольшая доля назначений приходилась на торасемид. Однако каждый четвертый пациент получал тиазидные и тиазидоподобные диуретики в дозах, недостаточных для лечения ХCH.

Среди тех, кому были назначены антагонисты минералокортикоидных рецепторов, основная масса пациентов получали спиронолактон, каждый шестой пациент - эплеренон (см. таблицу).

\section{Обсуждение}

Многие регистры показывают, что существуют значимые региональные различия в характеристике пациентов с ХCH, наблюдаемых в амбулаторном звене или госпитализированных, так же как в характере лечения и, соответственно, исходах у этих пациентов [7, 12].

Выявление «болевых точек», характерных для каждого конкретного региона, в котором проводится реструктуризация, может помочь

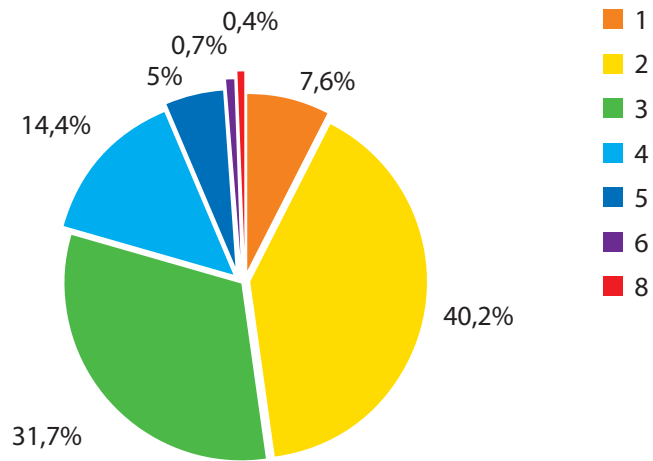

Рис. 1. Количество сопутствующих заболеваний

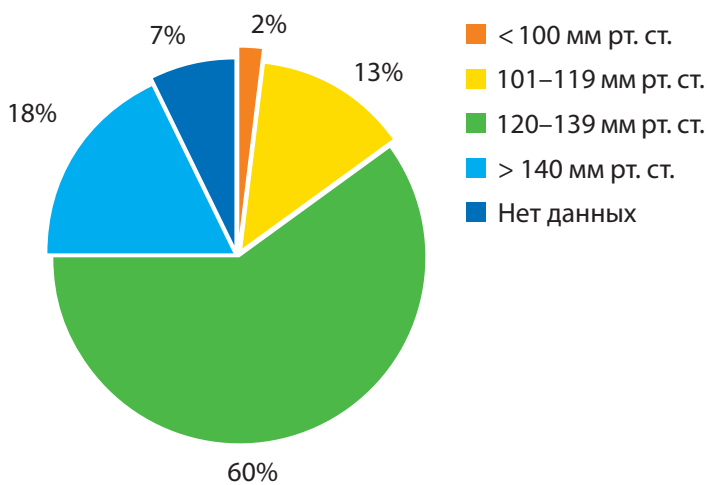

Рис. 2. Распределение пациентов с хронической сердечной недостаточностью по высоте артериального давления

значительно улучшить качество помощи и избежать ненужных или несвоевременных затрат. Характеризуя пациентов, которые наблюдаются в амбулаторном звене в Московской области, отметим: среди наших пациентов чаще встречалась артериальная гипертония (95,4 против 58\% в европейских странах), ишемическая болезнь сердца и перенесенный инфаркт миокарда (54 против $43 \%)$, несколько чаще наблюдался СД (37,9 против $31,9 \%)$, с одинаковой частотой отмечались перенесенный инсульт, наличие ХБП, фибрилляция предсердий [7].

Сравнивая полученные нами данные по Московской области с опубликованными результатами по Нижнему Новгороду, следует отметить как ряд схожих черт, например, высокую частоту артериальной гипертонии, так и различия, например, в количестве случаев выявления СД [13-15]. К сожалению, в цитируемых работах отсутствуют подробные данные о характере проводимой терапии.

Многие пациенты имели несколько сердечно-сосудистых заболеваний, которые привели к развитию $\mathrm{XCH}$, а также сопутствующих 
Распределение пациентов с хронической сердечной недостаточностью по частоте назначения препаратов

\begin{tabular}{|c|c|}
\hline Международное непатентованное наименование & $\begin{array}{l}\text { Количество пациентов, } \\
\text { абс. (\%) }\end{array}$ \\
\hline \multicolumn{2}{|l|}{ Ингибиторы ангиотензинпревращающего фермента } \\
\hline Эналаприл & $79(46,5)$ \\
\hline Лизиноприл & $31(18,2)$ \\
\hline Периндоприл & $51(30,0)$ \\
\hline Рамиприл & $4(2,4)$ \\
\hline Фозиноприл & $5(2,9)$ \\
\hline \multicolumn{2}{|l|}{ Сартаны } \\
\hline Лозартан & $44(77,2)$ \\
\hline Валсартан & $8(14,0)$ \\
\hline Телмисартан & $2(3,5)$ \\
\hline Азилсартана медоксомил & $1(1,8)$ \\
\hline Олмесартана медоксомил & $1(1,8)$ \\
\hline Ирбесартан & $1(1,8)$ \\
\hline \multicolumn{2}{|l|}{ Бета-адреноблокаторы } \\
\hline Бисопролол & $172(76,1)$ \\
\hline Карведилол & $11(4,9)$ \\
\hline Метопролол & $40(17,7)$ \\
\hline Небиволол & $3(1,3)$ \\
\hline \multicolumn{2}{|l|}{ Диуретики } \\
\hline Торасемид & $98(67,0)$ \\
\hline Фуросемид & $12(8,2)$ \\
\hline Индапамид & $24(16,4)$ \\
\hline Гидрохлоротиазид & $15(10,3)$ \\
\hline \multicolumn{2}{|l|}{ Антагонисты минералокортикоидных рецепторов } \\
\hline Спиронолактон & $128(84,3)$ \\
\hline Эплеренон & $24(15,8)$ \\
\hline
\end{tabular}

состояний (см. рис. 1), что позволяет назвать полиморбидность характерной чертой для этой категории больных. Полученные нами данные в целом совпадают с данными, представленными по европейским странам [9]. Однако у наших пациентов существенно реже регистрировали частоту ХБП и анемии, при этом СД встречался чаще. Подчеркнем: наличие коморбидностей, и в частности ХБП, анемии и СД, имеет значимое неблагоприятное влияние на прогноз.

Если сравнивать полученные нами данные о структуре сердечно-сосудистых заболеваний у пациентов с ХCH с таковыми в Европе, следует подчеркнуть, что среди наших пациентов отмечается высокая частота артериальной гипертонии. В среднем по группе обследованных показатели АД (см. рис. 2) находились в нормальном пределе, однако более подробный анализ позволил установить, что нормальным этот уровень был лишь у $2 / 3$ пациентов, при этом показатели каждого пятого значительно превышали целевые значения, а низкий уровень АД наблюдался у очень небольшого числа пациентов. Это свидетельствует о том, что недостаточное количество тяжелых пациентов с ХCH попадают в круг внимания врачей, наблюдающих таких больных. Обращает также на себя внимание, что у 7\% пациентов вообще отсутствовали данные об уровне АД и лишь у $2 / 3$ пациентов была рассчитана СКФ.

Частота проведения эхокардиографии в Московской области достаточно высока и существенно превышает обычно публикуемые цифры. Однако отметим: основная масса пациентов имеет нормальную ФВ, и, соответственно, более тяжелые пациенты, для которых наилучшим образом разработаны рекомендации по тактике ведения, остаются вне поля зрения терапевтов и кардиологов. Так, при оценке характера назначаемой терапии в регионе Московской области невозможно не обратить внимание на несоответствие или неполное соответствие действующим рекомендациям. Доля пациентов, получающих рекомендованную терапию, значимо ниже, чем сообщается в международных, в частности европейских регистрах [16]. Согласно полученным нами данным, блокаторы РААС получали 80,4\% пациентов, бета-адреноблокаторы - 79,0\%, диуретики - 61,6\%, АМРК - 57,4\%. Крайне низким было назначение сердечных гликозидов - всего у 6,9\%. Дезагреганты получали 61,2\% пациентов, причем в ряде случаев они находились на двойной дезагрегантной терапии. Антикоагулянты получали все пациенты с фибрилляцией предсердий - 37,7\% (дозировки в большинстве случаев были правильными), статины - 70,3\%. При этом частота назначений бета-адреноблокаторов, иАПФ, диуретиков, сердечных гликозидов, ивабрадина была существенно ниже, чем об этом сообщают в контексте европейских стран, но 
отметим, что наши пациенты чаще получают нитраты и статины [16].

Обращает на себя внимание, что ряд используемых препаратов (телмисартан, азилсартан, олмесартан, ирбесартан, метопролола тартрат) в соответствии с утвержденными инструкциями не имеют зарегистрированного показания для лечения $\mathrm{XCH}$.

В исследовании A.P. Maggioni и соавт. [17] показано, что меньше трети пациентов получали целевые дозировки, предлагаемые действующими рекомендациями: $29,3 \%$ для иАПФ, $24,1 \%$ для блокаторов рецепторов ангиотензина II, 17,5\% для бета-блокаторов и $30 \%$ для АМКР. Как сообщали врачи, примерно у трети пациентов, не достигших целевых доз, они продолжали их титрацию, но относительно еще одной трети убедительного объяснения не представлено $(28,8 \%$ для иАПФ, $29,3 \%$ для блокаторов рецепторов ангиотензина II и 29,2\% для бета-блокаторов). Самый высокий уровень недостижения целевой дозировки в отсутствие веских причин зарегистрирован для АМКР - 46,9\% [17]. Подобные результаты отмечены в большинстве регистров или исследований по сердечной недостаточности [18-20].

Таким образом, в клинической практике действительно существует разрыв между используемыми дозами препаратов и рекомендованными на основании результатов рандомизированных клинических исследований. Это может быть обусловлено недостаточным опытом врачей, низким следованием клиническим рекомендациям, терапевтической инертностью в отношении «повышающего титрования» и др. Известно, что приверженность к исполнению рекомендаций недостаточно высокая среди не только пациентов, но и врачей. В этой связи представляется безусловно необходимой дополнительная подготовка врачей, как и повышение информированности о клинических рекомендациях. Следует также обращать внимание врачей на то, что применение основных препаратов, используемых для лечения $\mathrm{XCH}$, в дозировках, ниже рекомендованных, ассоциировано с большим риском госпитализации в связи с острой декомпенсацией ХСН [21]. При плохой приверженности врачей рекомендациям у пациентов с ХСН увеличивается риск смертельных осложнений и частоты госпитализаций в 2-2,5 раза [22].

\section{Заключение}

Проведенное исследование свидетельствует о том, что врачи уделяют недостаточное внимание группе тяжелых пациентов с ХСН. Назначение лекарственной терапии не вполне соответствует актуальным клиническим рекомендациям. Необходимо улучшить обучение врачей, наблюдающих пациентов с ХCH, с целью увеличения приверженности врачей к выполнению клинических рекомендаций в отношении обследования и лечения пациентов с данной патологией. (安)

\section{Дополнительная информация}

\section{Финансирование}

Работа выполнена по инициативе авторов без привлечения финансирования.

Конфликт интересов

Авторы декларируют отсутствие явных и потенциальных конфликтов интересов, связанных с содержанием настоящей статьи.

\begin{abstract}
Участие авторов
Оба автора внесли равный вклад в написание статьи. Оба автора прочли и одобрили финальную версию статьи перед публикацией, согласны нести ответственность за все аспекты работы и гарантируют, что ими надлежащим образом были рассмотрены и решены вопросы, связанные с точностью и добросовестностью всех частей работы.
\end{abstract}

\section{Литература / References}

1.Ponikowski P, Voors AA, Anker SD, Bueno H, Cleland JGF, Coats AJS, Falk V, González-Juanatey JR, Harjola VP, Jankowska EA, Jessup $M$, Linde $C$, Nihoyannopoulos $\mathrm{P}, \mathrm{Pa}$ rissis JT, Pieske B, Riley JP, Rosano GMC, Ruilope LM, Ruschitzka F, Rutten FH, van der Meer P; ESC Scientific Document Group. 2016 ESC Guidelines for the diagnosis and treatment of acute and chronic heart failure: The Task Force for the diagnosis and treatment of acute and chronic heart failure of the European Society of Cardiology (ESC) Developed with the special contribution of the Heart Failure Association (HFA) of the
ESC. Eur Heart J. 2016;37(27):2129-2200. doi: 10.1093/eurheartj/ehw128.

2. Мареев ВЮ, Фомин ИВ, Агеев ФТ, Беграмбекова ЮЛ, Васюк ЮА, Гарганеева АА, Гендлин ГЕ, Глезер МГ, Готье СВ, Довженко ТВ, Кобалава ЖД, Козиолова НА, Коротеев АВ, Мареев ЮВ, Овчинников АГ, Перепеч НБ, Тарловская ЕИ, Чесникова АИ, Шевченко АО, Арутюнов ГП, Беленков ЮН, Галявич АС, Гиляревский СР, Драпкина ОМ, Дупляков ДВ, Лопатин ЮМ, Ситникова МЮ, Скибицкий ВВ, Шляхто ЕВ. Клинические рекомендации ОССН - РКО - РНМОТ. Сердечная недостаточность: хроническая (ХСH) и острая де- компенсированная (ОДСН). Диагностика, профилактика и лечение. Кардиология. 2018;58(6S):8-158. doi: 10.18087/cardio.2475. [Mareev VYu, Fomin IV, Ageev FT, Begrambekova YuL, Vasyuk YuA, Garganeeva AA, Gendlin GE, Glezer MG, Gautier SV, Dovzhenko TV, Kobalava ZhD, Koziolova NA, Koroteev AV, Mareev YuV, Ovchinnikov AG, Perepech NB, Tarlovskaya El, Chesnikova Al, Shevchenko AO, Arutyunov GP, Belenkov YuN, Galyavich AS, Gilyarevsky SR, Drapkina OM, Duplyakov DV, Lopatin YuM, Sitnikova MYu, Skibitsky VV, Shlyakhto EV. [Russian Heart Failure Society, Russian Society of Cardiology. Russian Scientific Medical 
Society of Internal Medicine Guidelines for Heart failure: chronic (CHF) and acute decompensated (ADHF). Diagnosis, prevention and treatment]. Kardiologiia [Cardiology]. 2018;58(6S):8-158. Russian. doi: 10.18087/cardio.2475.]

3. Орлова ЯА, Ткачёва ОН, Арутюнов ГП, Котовская ЮВ, Васюк ЮА, Лопатин ЮМ, Мареев ВЮ, Мареев ЮВ, Стражеско ИД, Скворцов АА, Рунихина НК, Фролова ЕВ. Особенности диагностики и лечения хронической сердечной недостаточности у пациентов пожилого и старческого возраста. Мнение экспертов Общества специалистов по сердечной недостаточности, Российской ассоциации геронтологов и гериатров и Евразийской ассоциации терапевтов. Кардиология. 2018;58(12S):42-72. doi: 10.18087/cardio.2560. [Orlova IA, Tkacheva ON, Arutyunov GP, Kotovskaya YuV, Lopatin YuM, Mareev VYu, Mareev YuV, Skvortsov AA, Runihina NK, Frolova EV, Strazhesko ID. [Features of diagnostics and treatment of chronic heart failure in elderly and senile patients. Expert opinion of the Society of Experts in Heart Failure, Russian Association of Gerontologists, and Euroasian Association of Therapists]. Kardiologiia [Cardiology]. 2018;58(12S):42-72. Russian. doi: $10.18087 /$ cardio.2560.]

4.Беленков ЮН. Влияние специализированных форм активного амбулаторного ведения на функциональный статус, качество жизни и показатели гемодинамики больных с выраженной сердечной недостаточностью. Результаты Российской программы «ШАНС». Сердечная недостаточность. 2007;8(3):112-116. [Belenkov YuN. [Influence of specialized forms of active outpatient management on functional status, quality of life and hemodynamic parameters in patients with severe heart failure. Results of the Russian program "CHANCE"]. Serdechnaya Nedostatochnost' [Heart Failure]. 2007;8(3):112-116. Russian.]

5. Task force of the Hellenic Heart Failure Clinics Network. How to develop a national heart failure clinics network: a consensus document of the Hellenic Heart Failure Association. ESC Heart Fail. 2020;7(1):15-25. doi: 10.1002/ ehf2.12558.

6. Gandhi S, Mosleh W, Sharma UC, Demers C, Farkouh ME, Schwalm JD. Multidisciplinary Heart Failure Clinics Are Associated With Lower Heart Failure Hospitalization and Mortality: Systematic Review and Meta-analysis. Can J Cardiol. 2017;33(10):1237-1244. doi: 10.1016/j.cjca.2017.05.011.

7. Komajda M, Follath F, Swedberg K, Cleland J, Aguilar JC, Cohen-Solal A, Dietz R, Gavazzi A, Van Gilst WH, Hobbs R, Korewicki J, Madeira HC, Moiseyev VS, Preda I, Widimsky J, Freemantle N, Eastaugh J, Mason J; Study Group on Diagnosis of the Working Group on Heart Failure of the European Society of Cardiology. The EuroHeart Failure Survey programme - a survey on the quality of care among patients with heart failure in Europe. Part 2: treatment. Eur Heart J. 2003;24(5):464-474. doi: 10.1016/ s0195-668x(02)00700-5.

8. Виноградова НГ, Жиркова ММ, Фомин ИВ, Поляков ДС. Эффективность лечения хронической сердечной недостаточности на амбулаторном этапе в условиях городского центра ХCH. Сердечная недостаточность. 2017;18(4):270-278. doi: 10.18087/ RHFJ.2017.4.2355. [Vinogradova NG, Zhirkova MM, Fomin IV, Polyakov DS. [Efficacy of therapy for chronic heart failure at the outpatient stage in the conditions of a Municipal Center for CHF]. Serdechnaya Nedostatochnost' [Heart Failure]. 2017;18(4):270-278. Russian. doi: 10.18087/RHFJ.2017.4.2355.]

9. van Deursen VM, Urso R, Laroche C, Damman K, Dahlström U, Tavazzi L, Maggioni AP, Voors AA. Co-morbidities in patients with heart failure: an analysis of the European Heart Failure Pilot Survey. Eur J Heart Fail. 2014;16(1):103-111. doi: 10.1002/ejhf.30.

10. Kristensen SL, Martinez F, Jhund PS, Arango JL, Bĕlohlávek J, Boytsov S, Cabrera W, Gomez E, Hagège AA, Huang $J$, Kiatchoosakun $S$, Kim KS, Mendoza I, Senni M, Squire IB, Vinereanu $D$, Wong RC, Gong J, Lefkowitz MP, Rizkala AR, Rouleau JL, Shi VC, Solomon SD, Swedberg K, Zile MR, Packer M, McMurray JJ. Geographic variations in the PARADIGM-HF heart failure trial. Eur Heart J. 2016;37(41):3167-3174. doi: 10.1093/eurheartj/ehw226.

11.Управление Федеральной службы государственной статистики по г. Москве и Московской области. Московская область в цифрах: Статистический сборник [Интернет]. М.; 2020. 390 с. Доступно на: https:// mosstat.gks.ru/storage/mediabank/MTaTDg7F/\%D0\%9C\%D0\%9E\%20\%D0\%B2\%20 \%D1\%86\%D0\%B8\%D1\%84\%D1\%80\%D0\%B 0\%D1\%85\%202019.pdf. [Department of the Federal State Statistics Service for Moscow and the Moscow Region. Moscow Region in figures. Statistical collection [Internet]. Moscow; 2020. 390 p. Russian. Available from: https:// mosstat.gks.ru/storage/mediabank/MTaTDg7F/\%D0\%9C\%D0\%9E\%20\%D0\%B2\%20\%D1 \%86\%D0\%B8\%D1\%84\%D 1\%80\%D0\%B0 \%D1\%85\%202019.pdf.]

12. Maggioni AP, Dahlström U, Filippatos G, Chioncel O, Crespo Leiro M, Drozdz J, Fruhwald F, Gullestad L, Logeart D, Fabbri G, Urso R, Metra $\mathrm{M}$, Parissis J, Persson H, Ponikowski P, Rauchhaus M, Voors AA, Nielsen OW, Zannad F, Tavazzi L; Heart Failure Association of the European Society of Cardiology (HFA). EURObservational Research Programme: regional differences and 1-year follow-up results of the Heart Failure Pilot Survey (ESC-HF Pilot). Eur J Heart Fail. 2013;15(7):808-817. doi: 10.1093/eurjhf/hft050.

13. Фомин И.В. Эпидемиология хронической сердечной недостаточности в Российской Фе- дерации. В: Агеев ФТ, ред. Хроническая сердечная недостаточность. М.: ГЭОТАР-Медиа; 2010. c. 7-77. [Fomin IV. [Epidemiology of chronic heart failure in the Russian Federation]. In: Ageev FT, editor. [Chronic heart failure]. Moscow: GEOTAR-Media; 2010. p. 7-77. Russian.]

14. Фомин ИВ. Хроническая сердечная недостаточность в Российской Федерации: что сегодня мы знаем и что должны делать. Российский кардиологический журнал. 2016;(8): 7-13. doi: 10.15829/1560-4071-2016-8-7-13. [Fomin IV. [Chronic heart failure in Russian Federation: what do we know and what to do]. Russian Journal of Cardiology. 2016;(8):7-13. Russian. doi: 10.15829/1560-4071-2016-8-7-13.]

15. Краием Н, Поляков ДС, Фомин ИВ, Виноградова НГ, Валикулова ФЮ, Вайсберг АР, Бадин ЮВ, Щербинина ЕВ. Динамика распространенности хронической сердечной недостаточности и сахарного диабета в Нижегородской области с 2000 по 2017 год. Кардиология. 2018;58(2S):25-32. doi: 10.18087/cardio.2439. [Kraiem N, Polyakov DS, Fomin IV, Vinogradova NG, Valikulova FYu, Vaysberg AR, Badin YuV, Shechrbinina EV. [Time-related changes in prevalence of chronic heart failure and diabetes mellitus in Nizhny Novgorod Region between 2000 and 2017]. Kardiologiia [Cardiology]. 2018;58(2S):25-32. Russian. doi: 10.18087/cardio.2439.]

16. Crespo-Leiro MG, Anker SD, Maggioni AP, Coats AJ, Filippatos G, Ruschitzka F, Ferrari R, Piepoli MF, Delgado Jimenez JF, Metra $\mathrm{M}$, Fonseca $\mathrm{C}$, Hradec J, Amir O, Logeart $\mathrm{D}$, Dahlström U, Merkely B, Drozdz J, Goncalvesova E, Hassanein M, Chioncel O, Lainscak M, Seferovic PM, Tousoulis D, Kavoliuniene A, Fruhwald F, Fazlibegovic E, Temizhan A, Gatzov $\mathrm{P}$, Erglis A, Laroche C, Mebazaa A; Heart Failure Association (HFA) of the European Society of Cardiology (ESC). European Society of Cardiology Heart Failure Long-Term Registry (ESC-HF-LT): 1-year follow-up outcomes and differences across regions. Eur J Heart Fail. 2016;18(6):613-625. doi: 10.1002/ejhf.566.

17. Maggioni AP, Anker SD, Dahlström U, Filippatos G, Ponikowski P, Zannad F, Amir O, Chioncel O, Leiro MC, Drozdz J, Erglis A, Fazlibegovic E, Fonseca C, Fruhwald F, Gatzov $\mathrm{P}$, Goncalvesova $\mathrm{E}$, Hassanein $\mathrm{M}$, Hradec J, Kavoliuniene A, Lainscak M, Logeart D, Merkely $B$, Metra $M$, Persson $H$, Seferovic $P$, Temizhan A, Tousoulis D, Tavazzi L; Heart Failure Association of the ESC. Are hospitalized or ambulatory patients with heart failure treated in accordance with European Society of Cardiology guidelines? Evidence from 12,440 patients of the ESC Heart Failure Long-Term Registry. Eur J Heart Fail. 2013;15(10):1173-1184. doi: 10.1093/eurjhf/hft134.

18. Maggioni AP, Dahlström U, Filippatos G, Chioncel O, Leiro MC, Drozdz J, Fruhwald F, Gullestad L, Logeart D, Metra M, Parissis J, Pers- 
son $\mathrm{H}$, Ponikowski $\mathrm{P}$, Rauchhaus $\mathrm{M}$, Voors $\mathrm{A}$, Nielsen OW, Zannad F, Tavazzi L; Heart Failure Association of ESC (HFA). EURObservational Research Programme: the Heart Failure Pilot Survey (ESC-HF Pilot). Eur J Heart Fail. 2010;12(10):1076-1084. doi: 10.1093/eurjhf/ hfq154.

19. Düngen HD, Apostolovic S, Inkrot S, Tahirovic E, Töpper A, Mehrhof F, Prettin C, Putnikovic B, Neskovic AN, Krotin M, Sakac D, Lainscak M, Edelmann F, Wachter R, Rau T, Eschenhagen T, Doehner W, Anker SD, Waagstein F, Herrmann-Lingen C, Gelbrich G, Dietz R; CIBIS-ELD investigators and Project Multicentre Trials in the Competence Network Heart Failure. Titra- tion to target dose of bisoprolol vs. carvedilol in elderly patients with heart failure: the CIBISELD trial. Eur J Heart Fail. 2011;13(6):670-680 doi: 10.1093/eurjhf/hfr020.

20. Cullington D, Goode KM, Clark AL, Cleland JG. Heart rate achieved or beta-blocker dose in patients with chronic heart failure: which is the better target? Eur J Heart Fail. 2012;14(7): 737-747. doi: 10.1093/eurjhf/hfs060.

21.Арутюнов АГ, Драгунов ДО, Арутюнов ГП, Соколова АВ. Влияние величины дозы основных препаратов на риск повторной госпитализации пациентов с хронической сердечной недостаточностью. Терапевтический архив. 2016;88(1):29-34. doi: 10.17116/ terarkh201688129-34. [Arutyunov AG, Dragunov DO, Arutyunov GP, Sokolova AV. [Impact of the dosing of basic drugs on the risk of rehospitalization in patients with chronic heart failure]. Therapeutic Archive. 2016;88(1):29-34. Russian. doi: 10.17116/terarkh201688129-34.]

22. Komajda M, Cowie MR, Tavazzi L, Ponikowski P, Anker SD, Filippatos GS; QUALIFY Investigators. Physicians' guideline adherence is associated with better prognosis in outpatients with heart failure with reduced ejection fraction: the QUALIFY international registry. Eur J Heart Fail. 2017;19(11):1414-1423. doi: 10.1002/ejhf.887.

\title{
Clinical characteristics and treatment of outpatients with chronic heart failure in the Moscow Region
}

\author{
T.K. Chernyavskaya' • M.G. Glezer²
}

Aim: To perform clinical characterization of patients with chronic heart failure (CHF) in the Moscow Region and to assess if their current treatments meet the current clinical guidelines.

Materials and methods: Based on the information submitted from 11 outpatient clinics in the Moscow Region in December 2019, we analyzed cluding their concomitant diseases, types of assessments and their results, as well as current treatments.

Results: The most common concomitant disease was arterial hypertension (95.1\% of the patients). $53.8 \%$ of the patients had previous myocardial infarction, $37.8 \%$, diabetes mellitus, and $34.6 \%$, atrial fibrillation. Chronic kidney disease was present in $18.5 \%$ of the patients, valvular heart disease in $11.9 \%$, and past stroke in $10.5 \%$. Of non-cardiovascular diseases, the most common were gastrointestinal disorders (25.2\%), chronic obstructive pulmonary disease or asthma (9.8\%), and anemia (5.2\%). Only $8 \%$ of the patients had one concomitant disease, whereas $72 \%$ had 2 to 3 diseases, and $20 \%$ had at least 4 concomitant diseases. Mean number of comorbidities per patient was 2.7. Echocardiography had been performed in $82.9 \%$ of the cases. Mean left ventricular ejection fraction was $51.0 \pm 10.11 \%$; in $11.5 \%$ of the patients it was $\leq 40 \%$. Glomerular filtration rate (GFR) was retrospective data on 286 patients with CHF, in-

calculated in $58.7 \%$ of the patients. $35.9 \%$ of the patients had a GFR of less than $60 \mathrm{~mL} / \mathrm{min} / 1.73 \mathrm{~m}^{2}$ in $3.6 \%$ it was $\leq 30 \mathrm{~mL} / \mathrm{min} / 1.73 \mathrm{~m}^{2} .83 .2 \%$ of the patients were treated with renin angiotensin aldosterone system blockers (angiotensin-converting enzyme inhibitors, angiotensin receptor antagonists, sacubitril/valsartan), $79.0 \%$ with beta-blockers, $53.1 \%$ with mineralocorticoid receptor antagonists. Glycosides had been administered to $6.9 \%$ of the patients, and diuretics, to $51.1 \%$. In most cases, the doses administered were below those recommended by the international clinical guidelines.

Conclusion: We have confirmed the need to increase the adherence of doctors to the clinical guidelines on assessment and management of CHF patients.

Key words: heart failure, clinical guidelines, hospitalization, disease-modifying agents, regional specifics

For citation: Chernyavskaya TK, Glezer MG. Clinical characteristics and treatment of outpatients with chronic heart failure in the Moscow Region. Almanac of Clinical Medicine. 2021;49(2):125-131. doi: 10.18786/2072-0505-2021-49-023.

Received 28 March 2021; revised 27 April 2021; accepted 30 April 2021; published online 20 May 2021

\section{Conflict of interests}

The authors declare no conflict of interests.

Authors' contributions

Both authors have equally contributed to the manuscript, have read and approved the final version of the manuscript before submission, agreed to be accountable for all aspects of the work in ensuring that questions related to the accuracy or integrity of any part of the work have been appropriately investigated and resolved.
Tat'yana K. Chernyavskaya - MD, PhD, Associate Professor, Deputy Director on Organizational and Methodological Work'

$\triangle 61 / 2$ Shchepkina ul., Moscow, 129110, Russian Federation. E-mail: corona1974@mail.ru

Maria G. Glezer - MD, PhD, Professor, Chair of Cardiology, Functional and Ultrasonic Diagnostics, Institute of Clinical Medicine named after N.V. Sklifosovskiy²; ORCID: https://orcid.org/0000 0002-0995-1924. E-mail: 287ast@mail.ru
'Moscow Regional Research and Clinical Institute (MONIKI); 61/2 Shchepkina ul., Moscow, 129110, Russian Federation

${ }^{2}$ I.M. Sechenov First Moscow State Medical University; 8/2 Trubetskaya ul., Moscow, 119991, Russian Federation 